\title{
Not All Negatives are Equal: Label-Aware Contrastive Loss for Fine-grained Text Classification
}

\author{
Varsha Suresh \\ Dept. of Computer Science \\ National University of Singapore \\ varshasuresheu. nus. edu
}

\author{
Desmond C. Ong \\ Dept. of Information Systems and Analytics \\ National University of Singapore, \\ \& Institute of High Performance \\ Computing, A*STAR \\ dco@ comp.nus.edu.sg
}

\begin{abstract}
Fine-grained classification involves dealing with datasets with larger number of classes with subtle differences between them. Guiding the model to focus on differentiating dimensions between these commonly confusable classes is key to improving performance on fine-grained tasks. In this work, we analyse the contrastive fine-tuning of pre-trained language models on two fine-grained text classification tasks, emotion classification and sentiment analysis. We adaptively embed class relationships into a contrastive objective function to help differently weigh the positives and negatives, and in particular, weighting closely confusable negatives more than less similar negative examples. We find that Label-aware Contrastive Loss outperforms previous contrastive methods, in the presence of larger number and/or more confusable classes, and helps models to produce output distributions that are more differentiated.
\end{abstract}

\section{Introduction}

Fine-grained classification involves distinguishing between classes that have subtle variations among them. For example, in image classification, we can classify birds from non-birds, or attempt a more fine-grained classification of bird species (Akata et al., 2015). In NLP, one example is sentiment analysis, where we could have a coarse positive/negative classification, or a fine-grained set of categories that differentiate "positive" and "very positive" (i.e., an ordinal scale), such as in Socher et al. (2013). Similarly, for emotion classification, we could try to classify a text into 4 to 6 emotions, or into much finer classifications of 27 (Demszky et al., 2020) or 32 (Rashkin et al., 2019) emotion categories. This involves distinguishing between some closely confusable pairs of emotions, such as "sad" and "devastated", or "furious" and "annoyed". Fine-grained classification tasks are challenging precisely due to the presence of class interference amongst closely confusable classes (Collins et al., 2018; Zhao et al., 2017).

The standard approach today to task classification involves using a pre-trained language model (e.g., BERT) which is fine-tuned on downstream tasks using a standard cross-entropy loss. However, this standard loss may not be the optimal manner in which to train fine-grained classification models. A simple counterexample is that cross-entropy loss treats misclassifications as nominal, not ordinal, so misclassifying a "positive" as a "very positive" is no worse (in terms of the loss) as "very negative". But even within nominal categories, misclassifying "annoyed" as "furious" is quite different from a misclassification of "joyful", as there are varying degrees of semantic similarity between nominal categories. Intuitively, we can try to improve model performance by modifying the loss to reflect the contrast between pairs of examples of the same or different classes. Such contrastive approaches are widely used in computer vision tasks for label-noise reduction, semi-supervised and selfsupervised learning tasks (Le-Khac et al., 2020). More recently in NLP, Gunel et al. (2021) used a supervised contrastive loss to improve fine-tuning performance of pre-trained language models in several few-shot learning scenarios.

In this work, we incorporate inter-class relationships into a Label-aware Contrastive Loss (LCL), which helps the model to differentiate the weights between different negative samples. At a high level, the model adaptively learns which pairs of classes are more similar, and which are more different. We use a dual-model approach where a weighting model learns the inter-label relationships that are used in the main embedding model's contrastive objective. We evaluate our approach on two popular tasks in NLP: emotion recognition (4 datasets to span both coarse- and fine-grained classification), and sentiment analysis (with a coarse and fine-grained version of the same dataset). We find 
that LCL outperforms existing contrastive learning losses, and performs comparably with the stateof-the-art. We supplement our findings with targeted experiments to provide evidence for boundary conditions-situations in which LCL should work best—and for how LCL affects model prediction confidence.

\section{Related Work}

\subsection{Fine-grained classification}

Fine-grained classification is a popular problem in image classification, including tasks like distinguishing between different animal species (Wei et al., 2019; Zhao et al., 2017). We note that in NLP, "fine-grained" is commonly used when analysing different granularities of text, such as character-, word- and span-level information (Zirn et al., 2011; Da San Martino et al., 2019; Liu et al., 2020). In this work, we use fine-grained classification to refer to the nature of labels associated with the task.

Fine-grained classification tasks involve finding subtle differences to distinguish between close classes. For instance, "coarse" sentiment classification involves distinguishing negative and positive sentiments in text, and fine-grained sentiment classification involves further distinguishing the positive class into very positive and positive. This problem is challenging because the classes are semantically similar, which makes it difficult for the model to learn the labels (Collins et al., 2018).

Recent models have applied state-of-the-art attention mechanisms and multi-task learning to solve fine-grained sentiment classification. Balikas et al. (2017) performed fine-grained sentiment classification using a multi-task learning setup that performed both binary and fine-grained sentiment classification simultaneously. Yin et al. (2020) composed the sentiment semantics using an attention network to enhance BERT's pre-training objective, and showed improvement in a downstream fine-grained sentiment analysis task. Tian et al. (2020a) modified the pre-training objectives of language models to include more sentiment-specific tasks, such as sentiment word masking and sentiment word prediction, and showed improved performance in fine-grained sentiment analysis. These previous methods mostly focus on improving the pre-training of language models, or incorporating multiple task training; here, we focus on improving contrastive fine-tuning to solve fine-grained text classification.
Another important fine-grained classification task is that of emotion recognition. Traditionally, emotion recognition datasets have a small number of emotions (e.g., 4-7). Two recent datasets were proposed to address this issue: Rashkin et al. (2019) introduced Empathetic Dialogues, which contains text conversations labelled with 32 emotion labels, and Demszky et al. (2020) introduced GoEmotions, which contains Reddit comments labelled with 27 emotion labels. Recently, Suresh and Ong (2021) introduced a method to incorporate knowledge from emotion lexicons into an attention mechanism to improve fine-grained emotion classification on these two datasets. Khanpour and Caragea (2018) similarly used lexicon-based features to tackle fine-grained emotion recognition from online health posts. However, there is still much work to be done in fine-grained emotion classification, and it has important implications for designing empathetic agents and chatbots (Roller et al., 2021).

Finally, we note that fine-grained classification has also been explored in the context of entitytype classification (Ling and Weld, 2012; Jin et al., 2019). However, this task is generally multi-label in nature and is out of the scope of the current work.

\subsection{Contrastive learning}

Contrastive learning focuses on improving the ability of the model to differentiate a given data point from "positive" examples (points sharing the same label) and from "negative" examples (different labels). Contrastive learning has been widely used in computer vision, especially in self-supervised settings (Le-Khac et al., 2020; Chen et al., 2020) where such learning guides the model based on similarities between the latent representation of the samples. (Chen et al., 2020) introduced SimCLR, a simplified version of contrastive loss that does not use memory banks (Tian et al., 2020b; He et al., 2020; Misra and Maaten, 2020) or designated architectures (Bachman et al., 2019), and which achieves improved performance in both semi-supervised and self-supervised settings. SimCLR uses data augmentation to create "positive" examples that are similar to a given input. Khosla et al. (2020) extended SimCLR to also leverage label information: they include other training examples with the same label in the set of "positive" examples.

Contrastive loss has also been recently incorporated in both the pre-training and fine-tuning 
objectives of pre-trained language models. Selfsupervised contrastive loss has been used for pretraining language models such as BERT (Fang and Xie, 2020; Meng et al., 2021). Gunel et al. (2021) used a combination of cross entropy and supervised contrastive loss for fine-tuning pre-trained language models to improve performance in fewshot learning scenarios. Gao et al. (2021) used a contrastive objective to fine-tune pre-trained language models to obtain sentence embeddings, and achieved state-of-the-art performance in sentence similarity tasks. In our work, we aim to improve the fine-tuning objective of pre-trained language models for downstream tasks involving fine-grained classes.

\subsection{Other related work}

In addition to the above works, we mention other related references which used similar techniques. Dual-model approaches are used in tasks like knowledge distillation, where the knowledge from a larger teacher network is transferred to a lighter student model (Hinton et al., 2015; Kim and Rush, 2016; Sun et al., 2020, 2019; Li et al., 2020; Aguilar et al., 2020), however, these works are mainly focused on model compression. Dual-model strategies have also been widely used in label-noise representation learning in image classification tasks (Han et al., 2018; Wei et al., 2020; Lu et al., 2021; Feng et al., 2019) by updating each other with clean samples (the samples which have the lowest loss value in every iteration). However, the sample selection performed by these works assume that the noise rate in each dataset is known or needs to be estimated, which is not always possible.

Another set of works focus on sample reweighting to focus on select samples more. Plank et al. (2014) use inter-annotator agreement to guide the model's focus on samples that are harder to distinguish. Sample re-weighting is also widely used to reduce label noise. Although the majority of works in this area depend on a pre-determined weighting function, there are a few notable papers which automate this process by adaptively calculating weights: Chang et al. (2017) uses active learning to re-weight samples, while Ren et al. (2018) uses gradients to learn weights, however their performance drops with large number of classes (Song et al., 2020). Meta-Weight-Net uses a single-layer neural network to obtain the weights (Shu et al., 2019). These methods all require clean validation data to optimize their learning objective.

\section{Approach}

\subsection{Contrastive Loss}

A Contrastive Loss (CL) brings the latent representations of samples belonging to the same class closer together, by defining a set of positives (that should be closer) and negatives (that should be further apart). The type of positives and negatives vary and is dependent on the contrastive loss used. Throughout this section we denote the set of positives as $\mathcal{P}$ and set of negatives as $\mathcal{N}$. Let us also denote a batch of sample and label pairs as $\left\{x_{i}, y_{i}\right\}_{i \in I}$, where $I=\{1, \cdots, K\}$ is the indices of the samples and $K$ is the batch-size.

In the self-supervised version of contrastive loss (Chen et al., 2020), one applies augmentation to all $K$ samples to produce $K$ augmented datapoints. Therefore, the batch size becomes $2 K$ and $I=\{1, \cdots, 2 K\}$. The positive set for a given $x_{i}$ contains only one sample, the augmented version of $x_{i}$, and we denote its index as $g(i)$. The negative set would be the rest of the samples in the batch. The loss is defined as:

$$
L_{\text {self }}=\sum_{i=1}^{2 K}-\log \frac{\exp \left(h_{i} \cdot h_{g(i)} / \tau\right)}{\sum_{k \in \mathcal{I} / i} \exp \left(h_{i} \cdot h_{k} / \tau\right)}
$$

where $\tau$ is the temperature hyper-parameter. Larger values of $\tau$ scale down the dot-products, creating more difficult comparisons. $h_{i}$ is the normalised representation vector of $x_{i}$ obtained from an encoder $\Phi$.

Khosla et al. (2020) extended the above loss to a Supervised Contrastive Loss (SCL) by including the samples belonging to the same class as $x_{i}$ in its positive set. The positive set is given by $\mathcal{P}=$ $\left\{p: p \in I, y_{p}=y_{i} \wedge p \neq i\right\}$, with size $|\mathcal{P}|$. The supervised contrastive loss is given by:

$$
L_{S C L}=\sum_{i=1}^{2 K} \frac{-1}{|\mathcal{P}|} \sum_{p \in \mathcal{P}} \log \frac{\exp \left(h_{i} \cdot h_{p} / \tau\right)}{\sum_{k \in \mathcal{I} / i} \exp \left(h_{i} \cdot h_{k} / \tau\right)}
$$

\subsection{Label-aware Contrastive Loss}

In our work, we introduce relationships between class labels to adaptively distinguish between the negative examples. From Eqn. 2 we can see that Supervised Contrastive Loss weights all positive 


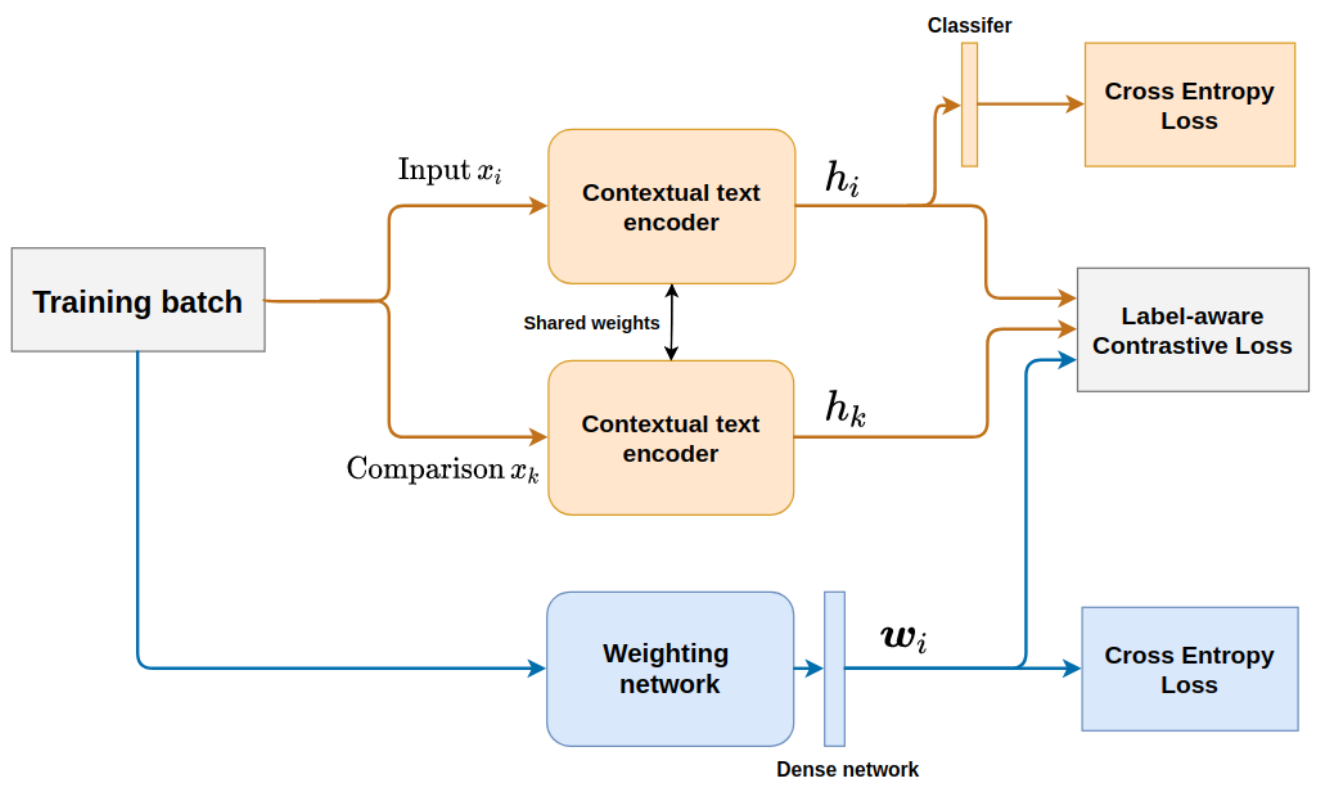

Figure 1: Illustration of training strategy used in our Label-aware Contrastive Loss approach. The encoder network is in orange and the the weighting network is indicated in blue. In the encoder network, every sample from the training batch is compared against every other sample in the Label-aware Contrastive Loss function. Note that at testing time, only the contextual encoder is used.

and negative samples equally to the current sample $x_{i}$. But not all negatives are equal. In certain fine-grained text classification tasks, we have semantically-similar labels with more subtle differences, and are thus more confusable. For example, "sad" and "devastated" are semantically closer emotion categories than "sad" and "happy". Thus, our goal was to introduce a method for adaptively weighting a given input's positive/negative samples based on the label-relationships between them, thereby helping the model differentiate the more difficult negatives.

We propose Label-aware Contrastive Loss (LCL) which adapts Contrastive Loss for fine-grained classification tasks by incorporating inter labelrelationships. For the positive set, we follow (Khosla et al., 2020; Gunel et al., 2021) where $\mathcal{P}$ of a given sample contains the augmented sample and samples within the same class. We utilise a weighting vector $\boldsymbol{w}_{\boldsymbol{i}} \in \mathbb{R}^{C}$ where $C$ is total number of classes to weight the pair-wise similarity values of the supervised contrastive loss defined in Eq. 2. Our adapted loss function for each entry $i$ and total across the batch is:

$$
\mathcal{L}_{i}=\sum_{p \in \mathcal{P}} \log \frac{w_{i, y_{i}} \cdot \exp \left(h_{i} \cdot h_{p} / \tau\right)}{\sum_{k \in \mathcal{I} \backslash i} w_{i, y_{k}} \cdot \exp \left(h_{i} \cdot h_{k} / \tau\right)}
$$

$$
L_{L C L}=\sum_{i=1}^{2 K} \frac{-1}{|\mathcal{P}|} \mathcal{L}_{i}
$$

Here, $w_{i, y_{k}}$ indicates the relationship between an input $x_{i}$ and a label $y_{k}$. Just as in the previous losses, $h_{i} \in \mathbb{R}^{d}$ is the output representation of the encoder for $x_{i}$. We normalise $h_{i}$ for the similarity comparison, similar to Chen et al. (2020).

In contrastive loss we want the weights of the positives to be higher and that of the negatives to be lower. However, we want to increase the weight of confusable negative labels relative to other negative labels. In our work, we aim to incorporate these inter-label relationships into the contrastive objective. To weigh each comparison sample differently, in addition to a primary encoder $\Phi$, we use a weighting network $\Psi$. We follow a dual-model strategy similar to co-teaching approaches (Han et al., 2018; Wei et al., 2020) where the weighting network is a second network that coordinates with the primary encoder. The input batch is fed into $\Psi$ and output is optimised using Cross-entropy loss $L_{w}$. The prediction probabilities obtained from the softmax layer, i.e. soft labels, is used to obtain confidence of the current sample, is given by:

$$
\boldsymbol{w}_{\boldsymbol{i}}=\frac{\exp \left(h_{i}\right)}{\sum_{c=1}^{C} \exp \left(h_{i}\right)}
$$


Here, $\boldsymbol{w}_{\boldsymbol{i}}=\left\{w_{i, c}\right\}_{c=1}^{C}$ where $C$ is the total number of classes. Each $w_{i, c}$ denotes the confidence of the weighting network that sample $x_{i}$ belongs to class $c$. When $\Psi$ is given a confusable sample, it will have higher scores for the classes that are more closely associated with the current sample. We hypothesize that incorporating these high values back into the negative comparison in the supervised contrastive loss of the primary encoder would steer the encoder toward finding more distinguishing patterns to differentiate between confusable samples.

Training setup: The output vector of the weighting network is optimized using a Cross Entropy Loss $L_{w}$, while the output of the encoder network is optimized by using a linear combination of $L_{L C L}$ and Cross Entropy Loss $L_{e}$. The encoder and weighting networks are jointly optimised using objective function $L_{f}$ :

$$
L_{f}=\alpha\left(L_{w}+L_{e}\right)+(1-\alpha) L_{L C L}
$$

Here, $\alpha$ is a tunable loss scaling factor similar to Gunel et al. (2021). We note that both the encoder and the weighting network are utilised during training, but in the testing phase, we use only the primary encoder network.

The overall training process is shown in Fig. 1. Each input training batch $I$ is passed to the encoder network $\Phi$ and the weighting network $\Psi$ simultaneously. Here, both these networks are initialised by a pre-trained language model and the [CLS] token of the last layer of $\Phi$ is the final representation $h_{i}$ which is used for computing $L_{L C L}$. For performing the classification, $h_{i}$ is projected down using the classifier and the output is optimised using cross-entropy loss $L_{e}$. The architecture of the weighting network was designed in the same way as the fine-tuning setup of the pre-trained language model of choice, and the weight vector $\boldsymbol{w}_{i}$ is the output probability vector obtained after the softmax projection.

\section{Experiments}

\subsection{Datasets}

We evaluate our approach using two tasks, Emotion Recognition and Sentiment Analysis. We choose these tasks as it helps demonstrate our model's performance in different types of inter-class relationships that exist in text classification. Specifically, in sentiment classification the classes are ordinal, whereas in emotion recognition the classes are nominal ${ }^{1}$.

For emotion recognition, we use the following 4 datasets, ordered in decreasing number of classes:

- Empathetic Dialogues (Rashkin et al., 2019)2: a dataset of two-way conversations between a speaker and listener, and labelled with 32 emotions. In this work, we only use the first turn of the conversation, which consists of the speaker describing an emotional incident. The train/validation/test split for the dataset is 19,533 / 2,770 / 2,547 samples respectively.

- GoEmotions (Demszky et al., 2020) ${ }^{3}$, a dataset of Reddit comments labelled with 27 emotions (we did not include samples with neutral label). The original dataset is multilabelled, i.e, some samples have more than one label. In this work, we use only the singlelabelled samples, which is $\sim 80 \%$ of the total data. The train/validation/test split of this dataset is $23,485 / 2,956 / 2,984$.

- ISEAR (International Survey on Emotion Antecedents and Reactions) (Scherer and Wallbott, 1994) ${ }^{4}$ contains sentences of emotion experiences labelled with one of 7 emotion categories. The train/validation/test split of the dataset is $4,599 / 1,533 / 1,534$.

- EmoInt (Mohammad and Bravo-Marquez, 2017) ${ }^{5}$ consists of tweets labelled with one of 4 emotion categories. The train/validation/test split of this dataset is 3,612 / 346 / 3,141.

For Sentiment Analysis, we use the 5-class and 2-class classification versions of the Standford Sentiment Treebank (Socher et al., 2013), which consists of movie reviews annotated for sentiment. The SST-5 has 5 classes (very negative, negative, neutral, positive, and very positive), while the SST-2 is only a binary (negative/positive) classification. The train/validation/test split for the SST-5 is $8,544 /$ 1,101 / 2,210, and for SST-2 is 6,920 / 872 / 1,821.

\footnotetext{
${ }^{1}$ Although there still may be underlying latent structure such that some classes may be semantically more similar than others, e.g., afraid vs. anxious vs. joyful.

${ }^{2}$ https://github.com/facebookresearch/ EmpatheticDialogues

${ }^{3}$ https://github.com/google-research/ google-research/tree/master/goemotions

${ }^{4}$ https: / / www . unige.ch/cisa/research/ materials-and-online-research/ research-material/

${ }^{5}$ http: / / saifmohammad.com/WebPages / EmotionIntensity-SharedTask.html
} 


\subsection{Implementation Details}

We initialised both the pre-trained encoder and weighting network using ELECTRA base $_{\text {be }}$ (electra-base-discriminator) from HuggingFace's Transformers library (Wolf et al., 2020), which consists of 12 Transformer layers with a hidden representation size of 768 . As is convention, we use the representation corresponding to the [CLS] token of the last layer as an input into the final classification layer (Clark et al., 2019). The classifier present in the primary encoder consists of a 2-layer dense network with the first layer having hidden size of 768 with a ReLU activation, followed by an output layer. The dropout was set to 0.1 .

Similar to previous research (Khosla et al., 2020; Gunel et al., 2021), we use data augmentation to generate positive samples. Here, we use synonym replacement where we substitute $30 \%$ of the words in the input text by replacing it with words with semantic similarity using WordNet dictionary (Miller, 1995). The coverage of the WordNet dictionary was $\sim 69 \%$ for EmpatheticDialogues, $\sim 69 \%$ for SST-2 and SST-5, $\sim 66 \%$ for ISEAR, $\sim 62 \%$ for EmoInt and $\sim 61 \%$ for GoEmotions. Previous research (Wei and Zou, 2019) have shown that synonym replacement works well as it could introduce new vocabulary words and help the model generalise. In addition, synonym replacement does not require an external model unlike other augmentation methods like back-translation.

For training, we used the Adam optimiser and early stopping based on performance on the validation set. We ran our models with 5 random seed settings and report the mean performance. More details regarding the hyper-parameter settings and computing infrastructure can be found in the Appendix. Source code is available at https: //github.com/varsha33/LCL_loss.

\subsection{Model comparisons and evaluation}

For the emotion classification task we calculate classification accuracy and F1 score, while for sentiment analysis we compare accuracy of sentencelevel sentiment classification. For both tasks, we compare LCL against the following baselines:

- Fine-tuning objectives: We compare against the standard Cross-entropy Loss, as well as Supervised Contrastive Loss (SCL) (Gunel et al., 2021). In both comparisons and in LCL, we use ELECTRA base $_{\text {as }}$ as the pre-trained language model.

- General pre-trained language models: For emotion classification, we also compare with

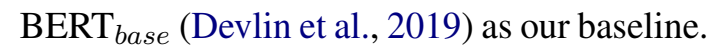
We use the same fine-tuning architecture as Devlin et al. (2019). For sentiment analysis, we compare against BERT base $_{\text {(SST-2 (Devlin }}$ et al., 2019) and SST-5 (Munikar et al., 2019)) and RoBERTa ${ }_{\text {base }}$ (Liu et al., 2019).

- Sentiment-specific language models: For sentiment analysis, we compare against SentiBERT (Yin et al., 2020), SentiLARE (Ke et al., 2020) and SKEP (Tian et al., 2020a), which are language models designed specifically for sentiment analysis and related tasks.

\section{Results and Discussion}

\subsection{Emotion Classification Performance}

For emotion classification we compared our proposed Label-aware Contrastive Loss (LCL) work with the standard training objective, i.e., crossentropy loss. We also compared with Gunel et al. (2021)'s formulation of Supervised Contrastive Loss (SCL), who used a linear combination of SCL and Cross-entropy loss for fine-tuning pre-trained language models (in contrast to the original SCL paper, Khosla et al., 2020, who used a two-stage training regime). For all fine-tuning objectives, we used ELECTRA $A_{\text {base }}$ as the pre-trained language model. To evaluate the approaches we use top-1 Accuracy and weighted macro F1-score.

As shown in Table 1, our LCL objective function improved classification performance compared to both SCL and cross-entropy loss, on both fine-grained emotion classification (32-class, LCL $>$ SCL, t-test on accuracy, $t=4.20, p=.007$, LCL $>$ CEL, $t=6.42, p<.001$; and 27-class classification; LCL $>$ SCL, $t=5.70, p<.001$, LCL $>$ CEL, $t=4.32, p=.002$ ), as well as coarsegrained emotion classification (7-class, LCL $>\mathrm{SCL}$, $t=7.39, p<.001, \mathrm{LCL}>\mathrm{CEL}, t=7.70, p<$ .001 ; and 4-class classification, LCL $>$ SCL, $t=$ $5.34, p<.001$, LCL $>$ CEL $, t=2.25, p=.078$ not significant). The consistent improved performance of LCL is in contrast to SCL, which did not outperform standard cross-entropy loss, (all $p>.05$, with SCL in fact performing worse than CEL on ISEAR, $t=3.34, p=.02$ ). These results suggest that incorporating class relationships into 


\begin{tabular}{|c|c|c|c|c|c|c|c|c|}
\hline \multirow[t]{2}{*}{$\begin{array}{l}\text { Dataset: } \\
\text { Number of classes: }\end{array}$} & \multicolumn{2}{|c|}{$\begin{array}{l}\text { Empathetic Dialogues } \\
32\end{array}$} & \multicolumn{2}{|c|}{$\begin{array}{c}\text { GoEmotions } \\
27\end{array}$} & \multicolumn{2}{|c|}{$\begin{array}{c}\text { ISEAR } \\
7\end{array}$} & \multicolumn{2}{|c|}{$\begin{array}{c}\text { EmoInt } \\
4\end{array}$} \\
\hline & Acc / \% & $\mathrm{F} 1$ & Acc / \% & F1 & Acc $/ \%$ & F1 & Acc / \% & $\mathrm{F} 1$ \\
\hline BERT $_{\text {base }}$ & $55.8(0.8)$ & $54.4(1.2)$ & $64.1(0.5)$ & $63.0(0.9)$ & $69.2(0.3)$ & $69.3(0.1)$ & $85.0(0.6)$ & $85.0(0.6)$ \\
\hline ELECTRA $_{\text {base }}+$ Cross-Entropy Loss & $58.3(0.5)$ & $56.8(0.5)$ & $64.8(0.3)$ & $63.9(0.4)$ & $71.4(0.2)$ & $71.4(0.2)$ & $85.5(0.9)$ & $85.5(0.9)$ \\
\hline ELECTRA $_{\text {base }}+$ SCL (Gunel et al., 2021) & $58.5(0.7)$ & $57.0(0.9)$ & $64.3(0.4)$ & $63.0(0.4)$ & $70.5(0.5)$ & $70.5(0.6)$ & $85.7(0.2)$ & $85.8(0.2)$ \\
\hline ELECTRA $_{\text {base }}+$ LCL & $60.1(0.3)$ & $59.1(0.3)$ & $65.5(0.2)$ & $64.8(0.2)$ & $72.4(0.2)$ & $72.4(0.2)$ & $86.6(0.3)$ & $86.6(0.3)$ \\
\hline
\end{tabular}

Table 1: Summary of results for fine-grained emotion recognition. We divide the table into fine-grained (left) and coarse-grained (right) emotion classification, based on the number of classes. We compare the results of an ELECTRA encoder trained with: a standard cross-entropy loss, a Supervised Contrastive Loss (SCL), and our proposed Label-aware Contrastive Loss (LCL). The results shown are averaged over 5 runs, with standard deviations in parenthesis.

the fine-tuning objective of pre-trained language models can improve classification accuracies.

\subsection{Sentiment Analysis Performance}

\begin{tabular}{|c|c|c|}
\hline & $\begin{array}{c}\text { SST-5 } \\
\text { Acc / } \%\end{array}$ & $\begin{array}{c}\text { SST-2 } \\
\text { Acc / } \%\end{array}$ \\
\hline BERT $_{\text {base }}$ (Munikar et al., 2019) & $53.2(-)$ & \\
\hline BERT $_{\text {base }}($ Devlin et al., 2019) & & 93.5(-) \\
\hline RoBERTa $_{\text {base }}$ (Liu et al., 2019) & $56.2(-)$ & $94.8(-)$ \\
\hline SentiBERT(Yin et al., 2020) & $57.8(-)$ & $94.7(-)$ \\
\hline SentiLARE(Ke et al., 2020) & $58.6(-)$ & \\
\hline SKEP (Tian et al., 2020a) & & $96.7(-)$ \\
\hline ELECTRA $_{\text {base }}$ (Clark et al., 2019) & & $93.4(-)$ \\
\hline$\overline{\mathrm{E}} \overline{\mathrm{L}} \overline{\mathrm{E}} \overline{\mathrm{T}} \overline{\mathrm{b}}_{\text {base }}$ (Our implemention $)$ & $5 \overline{7} . \overline{1}(1 . \overline{2})$ & $\overline{9} \overline{4} . \overline{4}(0.3)$ \\
\hline ELECTRA $_{\text {base }}+$ SCL (Gunel et al., 2021) & $57.4(0.6)$ & $94.3(0.2)$ \\
\hline ELECTRA $_{\text {base }}+$ LCL (Ours) & $58.5(0.2)$ & $94.5(0.1)$ \\
\hline
\end{tabular}

Table 2: Summary of results for fine-grained (5-class) and coarse-grained (2-class) sentiment analysis. The results shown are averaged over 5 runs, with standard deviations in parenthesis.

For sentiment analysis, we used the sentence inputs from SST-5 and SST-2. In addition to comparing LCL with varying fine-tuning objectives (crossentropy and SCL), we also compare against recent state-of-the-art works, focusing on pre-trained language models and pre-trained language models learnt specifically for sentiment classification such as SentiBERT (Yin et al., 2020), SentiLARE (Ke et al., 2020), and SKEP (Tian et al., 2020a). To ensure a fair comparison, we use the base version of the pre-trained language models unless mentioned otherwise. To evaluate, we use top-1 Accuracy.

From the results in Table 2, in the case of SST-5, our LCL objective showed improved classification performance compared to SCL $(t=$ $3.61, p=.01$ ), and standard cross-entropy loss (SST-5: $t=2.40, p=.069$, although this is not significant due to high SD in CEL performance). Our LCL-fine-tuned model also achieves a performance comparable to the state-of-the-art performance of SentiLARE, although not statistically different $(p=.77)$. On SST-2, our LCL performance gains compared to cross-entropy and SCL are far more modest (neither were statistically significant; $p=.78$ and $p=.32$ respectively), and it performs comparably to previous SOTA pre-trained models, although it does not do as well as SKEP $(p<.001)$. We provide two possible reasons: one, there is already very high performance (e.g. $94 \%$ accuracies) on this binary classification task, which makes it difficult to get clear consistent improvements. Second and more importantly, we designed LCL to increase inter-class contrast, and so our method should work better for higher number of classification, compared to binary classification. Indeed, we see that LCL's improvements are much stronger and consistent on the fine-grained (5-class) sentiment classification task.

\subsection{Case Study: Varying number of classes}

We designed LCL to increase inter-class contrast, and we see marked improvements for all the tasks studied except for the 2-class (SST-2) classification. We hypothesized that LCL should do better with an increasing number of classes, but unfortunately it is difficult to draw that inference from Tables 1 and 2 as each dataset only provides one datapoint about number of classes, and there are also differences across datasets which is difficult to control for. Thus, in this experiment, we used the dataset with the largest number of emotion classes, Empathetic Dialogues (with 32-classes), and subsampled some fraction of emotion classes from this dataset to create "mini-datasets" of differing number of emotion classes. This allows us to systematically vary the number of classes that our LCL-tuned model has to learn to classify, and examine the performance of the model. We predict that LCL will have a greater contribution to performance when (i) the number of classes is larger, and (ii) the classes are more confusable. 


\begin{tabular}{lccc|c|cccc} 
Number of classes: & $\mathbf{3 2}$ & $\mathbf{1 6}$ & $\mathbf{8}$ & 4-easy & 4hard-a & 4hard-b & 4hard-c & 4hard-d \\
\hline Cross-Entropy Loss & $58.1(0.7)$ & $68.8(0.4)$ & $78.0(0.6)$ & $\mathbf{8 9 . 2 ( 0 . 3 )}$ & $56.1(0.5)$ & $63.2(0.9)$ & $54.3(1.0)$ & $67.4(0.6)$ \\
Supervised Contrastive Loss & $58.6(0.5)$ & $67.9(0.6)$ & $77.0(0.8)$ & $88.8(0.5)$ & $55.4(0.5)$ & $63.7(1.1)$ & $53.3(0.8)$ & $68.1(0.7)$ \\
Label-aware Contrastive Loss & $\mathbf{6 0 . 1 ( 0 . 2 )}$ & $\mathbf{6 9 . 6 ( 0 . 5 )}$ & $\mathbf{7 8 . 7}(\mathbf{0 . 4})$ & $88.8(0.6)$ & $\mathbf{5 7 . 5}(\mathbf{0 . 7})$ & $\mathbf{6 4 . 2}(\mathbf{0 . 7})$ & $\mathbf{5 5 . 6}(\mathbf{0 . 6})$ & $\mathbf{6 9 . 5}(\mathbf{0 . 5})$
\end{tabular}

Table 3: Case study using class subsets of EmpatheticDialogues. For brevity, we only report accuracy scores. Column headers give the number of class labels in that comparison. 4-easy denotes a coarse-grained set of four emotions that are more easily distinguishable (on which we predicted that LCL would not add much), while the 4hard sets denote fine-grained sets of four emotions that are semantically more similar. Results shown are averaged over 10 runs, with standard deviations in parentheses.

The full dataset has 32-classes. We randomly sampled a partition of 16 emotions $^{2}$, and 8 emotions $^{3}$. We also created several subsets of 4emotions. We designed a "4-easy" with 4 widely separated emotion classes (4-easy: \{Angry, Afraid, Joyful, $S a d\}$ ) which are the same classes as EmoInt and comprise a subset of Ekman (1999)'s list of six "basic" emotions. (We predicted that LCL would not perform too well on this easy subset).

We adopted a data-driven approach to pick the "hard" subsets by picking the most-confusable sets of 4 emotions. First, we trained a standard crossentropy loss model (similar to our weighting network in LCL in Fig.1), to obtain the 32-by-32 confusion matrix, which gives us an estimate of how confusable each pair of classes is. We exhaustively enumerated all 35,960 (32-choose-4) 4-class combinations: For each combination we extracted the corresponding $4 \times 4$ sub-matrix of the 32-by32 confusion matrix, and calculated the sum of the off-diagonal elements of the $4 \times 4$ sub-matrix. The highest confusable combination of emotions was (4-hard-a: Anxious, Apprehensive, Afraid, Terrified $\})$. After excluding these emotions, the next-most confusable combinations were (4-hardb: \{Devastated, Nostalgic, Sad, Sentimental\}), (4-hard-c: \{Angry, Ashamed, Furious, Guilty\}), and (4-hard-d: \{Anticipating, Excited, Hopeful, Guilty\}). We predicted that for all of these "hard" sets that contain confusable emotions, LCL should outperform the other methods.

The results from this case study are given in Table 3. For the 32,16, and 8-class classification, as we predicted, we see a robust and consistent improvement of our proposed LCL over SCL and cross-entropy loss (16 classes: LCL $>\mathrm{SCL}$, $t=6.28, p<.001 ; \mathrm{LCL}>\mathrm{CEL}, t=3.82, p=$

\footnotetext{
${ }^{2}\{$ Afraid, Angry, Annoyed, Anxious, Confident, Disappointed, Disgusted, Excited, Grateful, Hopeful, Impressed, Lonely, Proud, Sad, Surprised, Terrified

${ }^{3}$ \{Angry, Afraid, Ashamed, Disgusted, Guilty, Proud, Sad, Surprised $\}$
}

$.001 ; 8$ classes: LCL $>$ SCL $, t=6.27, p<.001$; LCL $>$ CEL, $t=3.16, p=.007)$. For the easy 4-class classification where the classes are conceptually "far apart", and hence, contrastive learning should not add much, we see that all three methods perform identically well $(p>.15)$. But when we consider the more difficult 4-class classifications where the classes are much more conceptually similar, then LCL outperforms the other two methods by a statistically-significant margin (all $p$ 's $<.05$ except for LCL and SCL in 4-hard-b because of the high SD's in that comparison). Thus, our results provide evidence that LCL is an effective finetuning strategy, especially when there are a large number of highly-similar classes.

\subsection{Quantifying model confidence}

Finally, we wanted to try to quantify the intuition that LCL helps to reduce the confusion among confusable classes. Beyond looking at the top-1 accuracy, we turned to the distribution of prediction scores among the different emotion classes. If LCL helps the model to better differentiate emotion classes, then we should also see this in the distribution of prediction scores for the different classes. For example, consider an example where devastated is the model's predicted label, and sad is a closely confusable class; if LCL helps to sharpen the model's ability to differentiate closely confusable classes, then the model's prediction score for devastated should also be much higher than that for sad. In general, we predict that LCL would result in more "peaky" distributions.

We propose to use information-theoretic entropy to quantify this. We predict that LCL would result in prediction score distributions with lower entropy, which correponds to more "peaky" distributions. For a data point $x_{i}$, let us denote the prediction score as $S \in \mathbb{R}^{C}$, where $C$ is the total number of class labels. We then take the top- $k$ prediction scores $S_{k}$ as the sub-vector of $S$ with the $k$-largest values (i.e., for $k=2, S_{k}$ would consist of the two 


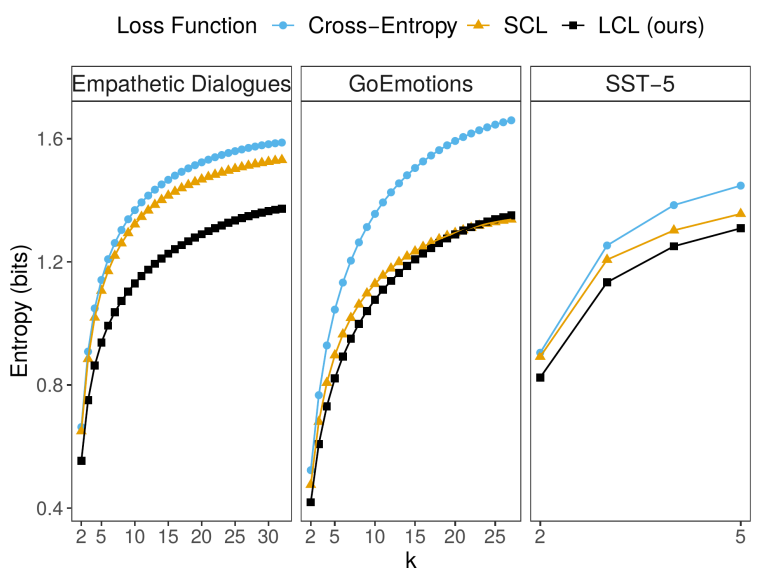

Figure 2: Averaged entropy of the prediction score distributions, for the top- $k$ choices. Here, decreasing entropy carries the intuition that the distribution is more "peaky", such that the model is less confused by close alternatives.

largest values in $S$ ). We normalize $S_{k}$ to sum to 1 , and then calculate the entropy:

$$
\text { Entropy }_{k}=-\sum_{k} s_{k} \cdot \log _{2}\left(s_{k}\right)
$$

In Figure 2, we present the averaged entropy of our model's prediction scores, plotted against $k$ for the fine-grained emotion classification (Empathetic Dialogues and GoEmotion) and fine-grained sentiment analysis (SST-5). For Empathetic Dialogues, we see that LCL produces distributions with far lower entropies, compared to cross-entropy and $\mathrm{SCL}$, and this is true as we look across the top- $k$ classes. For GoEmotions, we see a slightly different pattern, where both SCL and LCL produce markedly less-entropic distributions compared to the vanilla cross-entropy loss, but there was not much difference between SCL and our LCL. Finally, for SST-5, which was the most fine-grained sentiment analysis task we looked at, we start to see the same pattern that LCL produces the lowest entropy distributions, but this inference is limited by the small domain of $k$.

This post-hoc analysis suggests that LCL helps the model to learn prediction distributions that are more confident. Note that this analysis looks at the confidence of the model's choice compared to the space of possible choices, and is independent of whether or not the predictions are correct (i.e., an inaccurate but confident model will also produce peaky, lower-entropic distributions), and so this result complements the other evaluation metrics used (accuracy and F1-scores).

\section{Conclusion}

In this paper we introduced a Label-aware Contrastive Loss that weights (negative) classes based on how closely confusable they are with the target class. Fine-tuning with LCL showed increased classification performance, especially in situations with (i) larger number of classes, and (ii) more confusable classes. LCL also seems to encourage the model to be more confident in its decisions.

We view our approach as just one way to instantiate the general idea of adaptively weighting different classes, and future work could explore other methods such as incorporating external knowledge about the class labels, or incorporating different distance metrics between different classes. We feel that this class of approaches are promising, as they exemplify the idea that not all negative classes are or should be treated equally.

\section{Acknowledgements}

This research is supported by the National Research Foundation, Singapore under its AI Singapore Program (AISG Award No: AISG2-RP-2020-016).

\section{References}

Gustavo Aguilar, Yuan Ling, Yu Zhang, Benjamin Yao, Xing Fan, and Chenlei Guo. 2020. Knowledge distillation from internal representations. In Proceedings of the AAAI Conference on Artificial Intelligence, volume 34 , pages 7350-7357.

Zeynep Akata, Scott Reed, Daniel Walter, Honglak Lee, and Bernt Schiele. 2015. Evaluation of output embeddings for fine-grained image classification. In Proceedings of the IEEE Conference on Computer Vision and Pattern Recognition, pages 2927-2936.

Philip Bachman, R Devon Hjelm, and William Buchwalter. 2019. Learning representations by maximizing mutual information across views. In Advances in Neural Information Processing Systems, volume 32.

Georgios Balikas, Simon Moura, and Massih-Reza Amini. 2017. Multitask learning for fine-grained twitter sentiment analysis. In Proceedings of the 40th international ACM SIGIR Conference on Research and Development in Information Retrieval, pages $1005-1008$.

Haw-Shiuan Chang, Erik Learned-Miller, and Andrew McCallum. 2017. Active Bias: Training more accurate neural networks by emphasizing high variance samples. In Proceedings of the 31st International Conference on Neural Information Processing Systems, pages 1003-1013. 
Ting Chen, Simon Kornblith, Mohammad Norouzi, and Geoffrey Hinton. 2020. A simple framework for contrastive learning of visual representations. In International Conference on Machine Learning, pages 1597-1607. PMLR.

Kevin Clark, Minh-Thang Luong, Quoc V Le, and Christopher D Manning. 2019. ELECTRA: Pretraining text encoders as discriminators rather than generators. In International Conference on Learning Representations.

Edward Collins, Nikolai Rozanov, and Bingbing Zhang. 2018. Evolutionary data measures: Understanding the difficulty of text classification tasks. In Proceedings of the 22nd Conference on Computational Natural Language Learning, pages 380-391, Brussels, Belgium. Association for Computational Linguistics.

Giovanni Da San Martino, Seunghak Yu, Alberto Barrón-Cedeño, Rostislav Petrov, and Preslav Nakov. 2019. Fine-grained analysis of propaganda in news article. In Proceedings of the 2019 Conference on Empirical Methods in Natural Language Processing and the 9th International Joint Conference on Natural Language Processing (EMNLPIJCNLP), pages 5636-5646, Hong Kong, China. Association for Computational Linguistics.

Dorottya Demszky, Dana Movshovitz-Attias, Jeongwoo Ko, Alan Cowen, Gaurav Nemade, and Sujith Ravi. 2020. GoEmotions: A dataset of fine-grained emotions. In Proceedings of the 58th Annual Meeting of the Association for Computational Linguistics, pages 4040-4054, Online. Association for Computational Linguistics.

Jacob Devlin, Ming-Wei Chang, Kenton Lee, and Kristina Toutanova. 2019. BERT: Pre-training of deep bidirectional transformers for language understanding. In Proceedings of the 2019 Conference of the North American Chapter of the Association for Computational Linguistics: Human Language Technologies, Volume 1 (Long and Short Papers), pages 4171-4186.

Paul Ekman. 1999. Basic Emotions. John Wiley \& Sons, Ltd.

Hongchao Fang and Pengtao Xie. 2020. CERT: Contrastive self-supervised learning for language understanding. arXiv preprint arXiv:2005.12766.

Jiazhan Feng, Chongyang Tao, Wei Wu, Yansong Feng, Dongyan Zhao, and Rui Yan. 2019. Learning a matching model with co-teaching for multi-turn response selection in retrieval-based dialogue systems. In Proceedings of the 57th Annual Meeting of the Association for Computational Linguistics, pages 3805-3815.

Tianyu Gao, Xingcheng Yao, and Danqi Chen. 2021 SimCSE: Simple contrastive learning of sentence embeddings. arXiv preprint arXiv:2104.08821.
Beliz Gunel, Jingfei Du, Alexis Conneau, and Veselin Stoyanov. 2021. Supervised contrastive learning for pre-trained language model fine-tuning. In International Conference on Learning Representations.

Bo Han, Quanming Yao, Xingrui Yu, Gang Niu, Miao $\mathrm{Xu}$, Weihua Hu, Ivor Tsang, and Masashi Sugiyama. 2018. Co-teaching: Robust training of deep neural networks with extremely noisy labels. In $A d$ vances in Neural Information Processing Systems, volume 31 .

Kaiming He, Haoqi Fan, Yuxin Wu, Saining Xie, and Ross Girshick. 2020. Momentum contrast for unsupervised visual representation learning. In Proceedings of the IEEE/CVF Conference on Computer Vision and Pattern Recognition, pages 9729-9738.

Geoffrey Hinton, Oriol Vinyals, and Jeffrey Dean. 2015. Distilling the knowledge in a neural network. In NIPS Deep Learning and Representation Learning Workshop.

Hailong Jin, Lei Hou, Juanzi Li, and Tiansi Dong. 2019. Fine-grained entity typing via hierarchical multi graph convolutional networks. In Proceedings of the 2019 Conference on Empirical Methods in Natural Language Processing and the 9th International Joint Conference on Natural Language Processing (EMNLP-IJCNLP), pages 4969-4978, Hong Kong, China. Association for Computational Linguistics.

Pei Ke, Haozhe Ji, Siyang Liu, Xiaoyan Zhu, and Minlie Huang. 2020. SentiLARE: Sentiment-aware language representation learning with linguistic knowledge. In Proceedings of the 2020 Conference on Empirical Methods in Natural Language Processing $(E M N L P)$, Online. Association for Computational Linguistics.

Hamed Khanpour and Cornelia Caragea. 2018. Finegrained emotion detection in health-related online posts. In Proceedings of the 2018 Conference on Empirical Methods in Natural Language Processing, pages 1160-1166, Brussels, Belgium. Association for Computational Linguistics.

Prannay Khosla, Piotr Teterwak, Chen Wang, Aaron Sarna, Yonglong Tian, Phillip Isola, Aaron Maschinot, Ce Liu, and Dilip Krishnan. 2020. Supervised contrastive learning. Advances in Neural Information Processing Systems, 33.

Yoon Kim and Alexander M. Rush. 2016. Sequencelevel knowledge distillation. In Proceedings of the 2016 Conference on Empirical Methods in Natural Language Processing, pages 1317-1327, Austin, Texas. Association for Computational Linguistics.

Phuc H Le-Khac, Graham Healy, and Alan F Smeaton. 2020. Contrastive representation learning: A framework and review. IEEE Access. 
Jianquan Li, Xiaokang Liu, Honghong Zhao, Ruifeng $\mathrm{Xu}$, Min Yang, and Yaohong Jin. 2020. BERTEMD: Many-to-many layer mapping for BERT compression with earth mover's distance. In Proceedings of the 2020 Conference on Empirical Methods in Natural Language Processing (EMNLP), pages 3009-3018, Online. Association for Computational Linguistics.

Xiao Ling and Daniel Weld. 2012. Fine-grained entity recognition. In Proceedings of the AAAI Conference on Artificial Intelligence, volume 26.

Yinhan Liu, Myle Ott, Naman Goyal, Jingfei Du, Mandar Joshi, Danqi Chen, Omer Levy, Mike Lewis, Luke Zettlemoyer, and Veselin Stoyanov. 2019. RoBERTa: A robustly optimized bert pretraining approach. arXiv preprint arXiv:1907.11692.

Zhenghao Liu, Chenyan Xiong, Maosong Sun, and Zhiyuan Liu. 2020. Fine-grained fact verification with kernel graph attention network. In Proceedings of the 58th Annual Meeting of the Association for Computational Linguistics, pages 7342-7351, Online. Association for Computational Linguistics.

Yangdi Lu, Yang Bo, and Wenbo He. 2021. Comatching: Combating noisy labels by augmentation anchoring. arXiv preprint arXiv:2103.12814.

Yu Meng, Chenyan Xiong, Payal Bajaj, Saurabh Tiwary, Paul Bennett, Jiawei Han, and Xia Song. 2021. Coco-lm: Correcting and contrasting text sequences for language model pretraining. arXiv preprint arXiv:2102.08473.

George A. Miller. 1995. WordNet: A Lexical Database for English. Communications of the ACM, 38(11):39-41.

Ishan Misra and Laurens van der Maaten. 2020. Selfsupervised learning of pretext-invariant representations. In Proceedings of the IEEE/CVF Conference on Computer Vision and Pattern Recognition, pages 6707-6717.

Saif Mohammad and Felipe Bravo-Marquez. 2017. WASSA-2017 shared task on emotion intensity. In Proceedings of the 8th Workshop on Computational Approaches to Subjectivity, Sentiment and Social Media Analysis, pages 34-49, Copenhagen, Denmark. Association for Computational Linguistics.

Manish Munikar, Sushil Shakya, and Aakash Shrestha. 2019. Fine-grained sentiment classification using bert. In 2019 Artificial Intelligence for Transforming Business and Society (AITB), volume 1, pages 1-5. IEEE.

Barbara Plank, Dirk Hovy, and Anders Søgaard. 2014. Learning part-of-speech taggers with inter-annotator agreement loss. In Proceedings of the 14th Conference of the European Chapter of the Association for Computational Linguistics, pages 742-751, Gothenburg, Sweden. Association for Computational Linguistics.
Hannah Rashkin, Eric Michael Smith, Margaret Li, and Y-Lan Boureau. 2019. Towards empathetic opendomain conversation models: A new benchmark and dataset. In Proceedings of the 57th Annual Meeting of the Association for Computational Linguistics, pages 5370-5381, Florence, Italy. Association for Computational Linguistics.

Mengye Ren, Wenyuan Zeng, Bin Yang, and Raquel Urtasun. 2018. Learning to reweight examples for robust deep learning. In International Conference on Machine Learning, pages 4334-4343. PMLR.

Stephen Roller, Emily Dinan, Naman Goyal, Da Ju, Mary Williamson, Yinhan Liu, Jing Xu, Myle Ott, Eric Michael Smith, Y-Lan Boureau, and Jason Weston. 2021. Recipes for building an open-domain chatbot. In Proceedings of the 16th Conference of the European Chapter of the Association for Computational Linguistics: Main Volume, pages 300-325, Online. Association for Computational Linguistics.

Klaus R Scherer and Harald G Wallbott. 1994. Evidence for universality and cultural variation of differential emotion response patterning. Journal of Personality and Social Psychology, 66(2):310.

Jun Shu, Qi Xie, Lixuan Yi, Qian Zhao, Sanping Zhou, Zongben $\mathrm{Xu}$, and Deyu Meng. 2019. MetaWeight-Net: Learning an explicit mapping for sample weighting. In Advances in Neural Information Processing Systems, volume 32.

Richard Socher, Alex Perelygin, Jean Wu, Jason Chuang, Christopher D. Manning, Andrew Ng, and Christopher Potts. 2013. Recursive deep models for semantic compositionality over a sentiment treebank. In Proceedings of the 2013 Conference on Empirical Methods in Natural Language Processing, Seattle, Washington, USA. Association for Computational Linguistics.

Hwanjun Song, Minseok Kim, Dongmin Park, Yooju Shin, and Jae-Gil Lee. 2020. Learning from noisy labels with deep neural networks: A survey. arXiv preprint arXiv:2007.08199.

Siqi Sun, Yu Cheng, Zhe Gan, and Jingjing Liu. 2019. Patient knowledge distillation for BERT model compression. In Proceedings of the 2019 Conference on Empirical Methods in Natural Language Processing and the 9th International Joint Conference on Natural Language Processing (EMNLP-IJCNLP), pages 4323-4332, Hong Kong, China. Association for Computational Linguistics.

Siqi Sun, Zhe Gan, Yuwei Fang, Yu Cheng, Shuohang Wang, and Jingjing Liu. 2020. Contrastive distillation on intermediate representations for language model compression. In Proceedings of the 2020 Conference on Empirical Methods in Natural Language Processing (EMNLP), pages 498-508, Online. Association for Computational Linguistics. 
Varsha Suresh and Desmond C. Ong. 2021. Using knowledge-embedded attention to augment pretrained language models for fine-grained emotion recognition. In Proceedings of the 9th International Conference on Affective Computing and Intelligent Interaction (ACII 2021).

Hao Tian, Can Gao, Xinyan Xiao, Hao Liu, Bolei He, Hua Wu, Haifeng Wang, and Feng Wu. 2020a. SKEP: Sentiment knowledge enhanced pre-training for sentiment analysis. In Proceedings of the 58th Annual Meeting of the Association for Computational Linguistics, pages 4067-4076, Online. Association for Computational Linguistics.

Yonglong Tian, Dilip Krishnan, and Phillip Isola. 2020b. Contrastive multiview coding. In Computer Vision-ECCV 2020: 16th European Conference, Glasgow, UK, August 23-28, 2020, Proceedings, Part XI 16, pages 776-794. Springer.

Hongxin Wei, Lei Feng, Xiangyu Chen, and Bo An 2020. Combating noisy labels by agreement: A joint training method with co-regularization. In Proceedings of the IEEE/CVF Conference on Computer Vision and Pattern Recognition, pages 13726-13735.

Jason Wei and Kai Zou. 2019. EDA: Easy data augmentation techniques for boosting performance on text classification tasks. In Proceedings of the 2019 Conference on Empirical Methods in Natural Language Processing and the 9th International Joint Conference on Natural Language Processing (EMNLP-IJCNLP), pages 6382-6388, Hong Kong, China. Association for Computational Linguistics.

Xiu-Shen Wei, Jianxin Wu, and Quan Cui. 2019. Deep learning for fine-grained image analysis: A survey. arXiv preprint arXiv:1907.03069.

Thomas Wolf, Lysandre Debut, Victor Sanh, Julien Chaumond, Clement Delangue, Anthony Moi, Pierric Cistac, Tim Rault, Remi Louf, Morgan Funtowicz, Joe Davison, Sam Shleifer, Patrick von Platen, Clara Ma, Yacine Jernite, Julien Plu, Canwen Xu, Teven Le Scao, Sylvain Gugger, Mariama Drame, Quentin Lhoest, and Alexander Rush. 2020. Transformers: State-of-the-art natural language processing. In Proceedings of the 2020 Conference on Empirical Methods in Natural Language Processing: System Demonstrations, pages 38-45, Online. Association for Computational Linguistics.

Da Yin, Tao Meng, and Kai-Wei Chang. 2020. SentiBERT: A transferable transformer-based architecture for compositional sentiment semantics. In Proceedings of the 58th Annual Meeting of the Association for Computational Linguistics, pages 36953706, Online. Association for Computational Linguistics.

Bo Zhao, Jiashi Feng, Xiao Wu, and Shuicheng Yan 2017. A survey on deep learning-based fine-grained object classification and semantic segmentation. International Journal of Automation and Computing, 14(2):119-135.
Cäcilia Zirn, Mathias Niepert, Heiner Stuckenschmidt, and Michael Strube. 2011. Fine-grained sentiment analysis with structural features. In Proceedings of 5th International Joint Conference on Natural Language Processing, pages 336-344, Chiang Mai, Thailand. Asian Federation of Natural Language Processing. 


\section{A Appendix}

\section{A.1 Evaluation metrics}

We use top-1 accuracy and weighted macro F1score. Weighted F1-score takes care of the imbalance in the label distribution and the equation for weighted macro $\mathrm{F} 1$-score is given by,

$$
\text { weighted } \mathrm{F} 1=2 \sum_{c} \frac{n_{c}}{N} \frac{\text { precision }_{c} \times \text { recall }_{c}}{\text { precision }_{c}+\text { recall }_{c}}
$$

where, $n_{c}$ is number of samples in class $c$ and $N$ is the total number of samples.

\section{B Experiment settings}

For fine-tuning pre-trained models using Labelaware Contrastive Loss (LCL), we use Adam optimiser with $\beta_{1}$ set to $0.9, \beta_{2}$ set to 0.999 and $\epsilon$ set to $1 \mathrm{e}-06$ with weight decay set to $1 \mathrm{e}-02$. We used manual search for hyper-parameter search and the best model was chosen based on the best top-1 accuracy yielded in the validation data. Learning rate was chosen from set $\{1 \mathrm{e}-05,2 \mathrm{e}-05,3 \mathrm{e}-05\}$, loss scaling factor $\alpha$ was chosen from $\{0.1,0.2, \cdots, 0.5\}$ and temperature parameter $\tau$ was chosen from the set $\{0.1,0.3,0.5\}$. The best parameter setting of LCL are as follows, for EmpatheticDialogues, EmoInt, SST-5, SST-2, GoEmotions learning rate was found to be $2 \mathrm{e}-05$ and for ISEAR it was found to be $3 \mathrm{e}-05$. The $\alpha$ setting was found to be 0.5 for EmpatheticDialogues, EmoInt, SST-5, SST-2, ISEAR and 0.1 for GoEmotions. For all datasets except SST-5 the temperature parameter was found to be 0.3 and for SST-5 it was found to be 0.1. Batch size was set to 10 for all the datasets, as we have one augmented sample for every input sample the effective batch-size becomes 20 .

For EmoInt the tweet data was cleaned using by removing non-ascii characters, letter repetitions and extra white-spaces. In addition, all the usermentions and links were replaced to unique identifiers. We ran all our experiments using machine equipped with a NVIDIA Tesla T4 GPU.

\section{B.1 Average runtime and parameters}

During training time, the number of parameters trainable parameters is the combined number of parameters of the primary encoder and the weighting network, in our case we use the base of ELECTRA for both which has $110 \mathrm{M}$ parameters. The average run-time of the model for one epoch was found to be $2.9 \mathrm{~min}$ for EmoInt , $5.2 \mathrm{~min}$ for ISEAR, 19.8 min for GoEmotions, 19.7 min for EmpatheticDialogues, $6.1 \mathrm{~min}$ for SST-2 and $8.2 \mathrm{~min}$ for SST-5.

\section{Validation performance}

The corresponding validation performance for the reported test results are provided for emotion classification task in Table 5 and sentiment analysis task in Table 4.

\begin{tabular}{lcc}
\hline & SST-2 & SST-5 \\
& Acc / \% & Acc / \% \\
\hline Cross-Entropy & $94.2(0.4)$ & $53.3(0.7)$ \\
SCL (Gunel et al., 2021) & $94.4(0.1)$ & $54.5(1.2)$ \\
LCL & $\mathbf{9 4 . 8}(\mathbf{0 . 2})$ & $\mathbf{5 5 . 4}(\mathbf{0 . 8})$ \\
\hline
\end{tabular}

Table 4: Summary of validation results for sentiment analysis task. The results shown are averaged over 5 runs and the standard deviation is provided in the brackets. 


\begin{tabular}{lcccc|cccc}
\hline Dataset : & \multicolumn{2}{c}{ Empathetic Dialogues } & \multicolumn{2}{c|}{ GoEmotions } & \multicolumn{2}{c}{ ISEAR } & \multicolumn{3}{c}{ EmoInt } \\
Number of classes : & \multicolumn{2}{c}{32} & \multicolumn{2}{c}{27} & \multicolumn{2}{c}{7} & \multicolumn{2}{c}{4} \\
& Acc / \% & F1 & Acc / \% & F1 & Acc / \% & F1 & Acc / \% & F1 \\
\hline Cross-Entropy & $59.0(0.2)$ & $58.1(0.4)$ & $66.2(0.2)$ & $65.3(0.3)$ & $71.7(0.4)$ & $71.7(0.4)$ & $87.1(1.0)$ & $87.1(1.0)$ \\
SCL (Gunel et al., 2021) & $58.9(0.7)$ & $57.8(0.8)$ & $64.9(0.3)$ & $63.7(0.3)$ & $72.2(0.7)$ & $72.2(0.7)$ & $87.9(0.5)$ & $87.9(0.5)$ \\
LCL & $\mathbf{6 0 . 3 ( 0 . 4 )}$ & $\mathbf{5 9 . 7 ( 0 . 4 )}$ & $\mathbf{6 6 . 0}(\mathbf{0 . 2})$ & $\mathbf{6 5 . 3 ( 0 . 2 )}$ & $\mathbf{7 2 . 6 ( 0 . 2 )}$ & $\mathbf{7 2 . 6 ( 0 . 2 )}$ & $\mathbf{8 8 . 8 ( 0 . 8 )}$ & $\mathbf{8 8 . 9}(\mathbf{0 . 8})$ \\
\hline
\end{tabular}

Table 5: Summary of validation results for emotion classification task. The results shown are averaged over 5 runs and the standard deviation is provided in the brackets. 